\title{
Aortic root fistula complicating Austrian syndrome
}

\author{
Ahmed Bakhit ${ }^{1}$, Ajay Kumar Mishra ${ }^{2}$, Khushal Choudhary ${ }^{3}$, Mohamed Khaled Soufi ${ }^{4}$ \\ ${ }^{1}$ Fellow in Department of Clinical Cardiology, Saint Vincent Hospital, Worcester, MA; ${ }^{2}$ Postgraduate in Internal \\ Medicine, Saint Vincent Hospital, Worcester, MA; ${ }^{3}$ Postgraduate in Internal Medicine, Roger Williams Medical Center, \\ Providence, RI; ${ }^{4}$ Department of Cardiology, University of Texas Medical Branch, Galveston, TX, USA
}

\begin{abstract}
Austrian syndrome occurs in $1.2 \%$ of all patients with pneumococcal infective endocarditis. It presents with the triad of meningitis, pneumonia, and endocarditis. It is commonly seen in elderly males with a history of alcohol abuse, an immunocompromised state, or recent valve surgery. We present a case of Austrian syndrome presenting with paravalvular complications in the form of aortic root fistula. In this report, we describe the second patient with the community-acquired, pneumococcal, native, aortic valve, endocarditis with Austrian syndrome complicated by the development of an aortic fistula.
\end{abstract}

\section{Introduction}

Pneumococcal endocarditis incidence has dramatically decreased since the introduction of penicillin [1]. The link between pneumococcal meningitis, endocarditis, and pneumonia was initially described by Austrian and this triad of clinical mani-

Correspondence: Dr. Ajay Kumar Mishra, Department of Internal Medicine, Saint Vincent Hospital, Worcester, MA 01608, USA.

Tel. +1.508.3635000. E-mail: Ajay.Mishra@stvincenthospital.com

Key words: Endocarditis; meningitis; pneumococcal pneumonia; complications.

Conflict of interest: Authors have no conflicts of interest to declare.

Ethical statement: The article does not contain the participation of any humanbeing and animal.

Contributions: All the authors played a significant role in the paper. All authors have seen the manuscript and agree to the content and data.

Received for publication: 4 March 2021.

21 April 2021.Accepted for publication:

${ }^{\circ}$ Copyright: the Author(s), 2021

Licensee PAGEPress, Italy

Monaldi Archives for Chest Disease 2021; 91:1834

doi: 10.4081/monaldi.2021.1834

This article is distributed under the terms of the Creative Commons Attribution Noncommercial License (by-nc 4.0) which permits any noncommercial use, distribution, and reproduction in any medium, provided the original author(s) and source are credited. festations secondary to Streptococcus Pneumoniae has been named Austrian syndrome [2]. We describe a patient with Austrian syndrome presenting with paravalvular complication.

\section{Case Report}

A 52-year-old male presented with high grade, intermittent, fever with chills, moderate, persistent, holo-cranial headache, and generalized malaise for 3 weeks.

Upon arrival to the emergency department, his temperature was $39.4^{\circ} \mathrm{C}$, his heart rate was 100 per minute and his blood pressure was $110 / 70 \mathrm{mmHg}$. On physical examination, he appeared lethargic, without any meningeal signs, any cranial nerve deficits, or motor weakness. His bilateral plantar reflexes were normal. Laboratory investigations showed a white blood count (WBC) of $11000 / \mathrm{ml}$ with a neutrophil percentage of $85.8 \%$. Cerebrospinal fluid (CSF) analysis showed elevated total protein of $>150 \mathrm{mg} / \mathrm{dL}$, low glucose of $<1 \mathrm{mg} / \mathrm{dL}$, and CSF WBC count of 54 cells $/ \mathrm{mm}^{3}$ suggestive of bacterial meningitis. Initially, he was started on vancomycin and ceftriaxone. Initially, his CSF gram stain was negative but eventually, his CSF and blood cultures showed Streptococcus pneumonia, and his antibiotic was switched to Penicillin G based on susceptibilities. His magnetic resonance imaging of the brain showed small foci in the left cerebellar hemisphere and left parietal lobe which was suggestive of acute or subacute infarcts (Figure 1 A1,A2,B1,B2). His EKG did not show any new PR prolongation and his transesophageal echocardiogram (TEE) showed severe aortic regurgitation with vegetation on the left coronary cusp of the aortic valve (Figure 1 D1,D2). Urgent aortic valve replacement was planned and his perioperative computed tomography of the heart with coronaries demonstrated an aortic root fistula (Figure 1C) and airspace disease in the right upper lobes conclusive of pneumonia, thus completing the triad of Austrian Syndrome. He underwent urgent aortic valve replacement. Following the initiation of antibiotics, and surgery he had significant clinical improvement. Repeat blood cultures were documented to be sterile. He was discharged in a stable condition with antibiotics for a total duration of 4 weeks.

\section{Discussion}

Pneumococcal endocarditis contributes to $<3 \%$ of community-acquired infective endocarditis and is associated with higher mortality [3]. And $<1 \%$ pneumococcal endocarditis patients have the classical triad of pneumococcal meningitis, endocarditis, and pneumonia [4]. Risk factors including advanced age, alcohol 

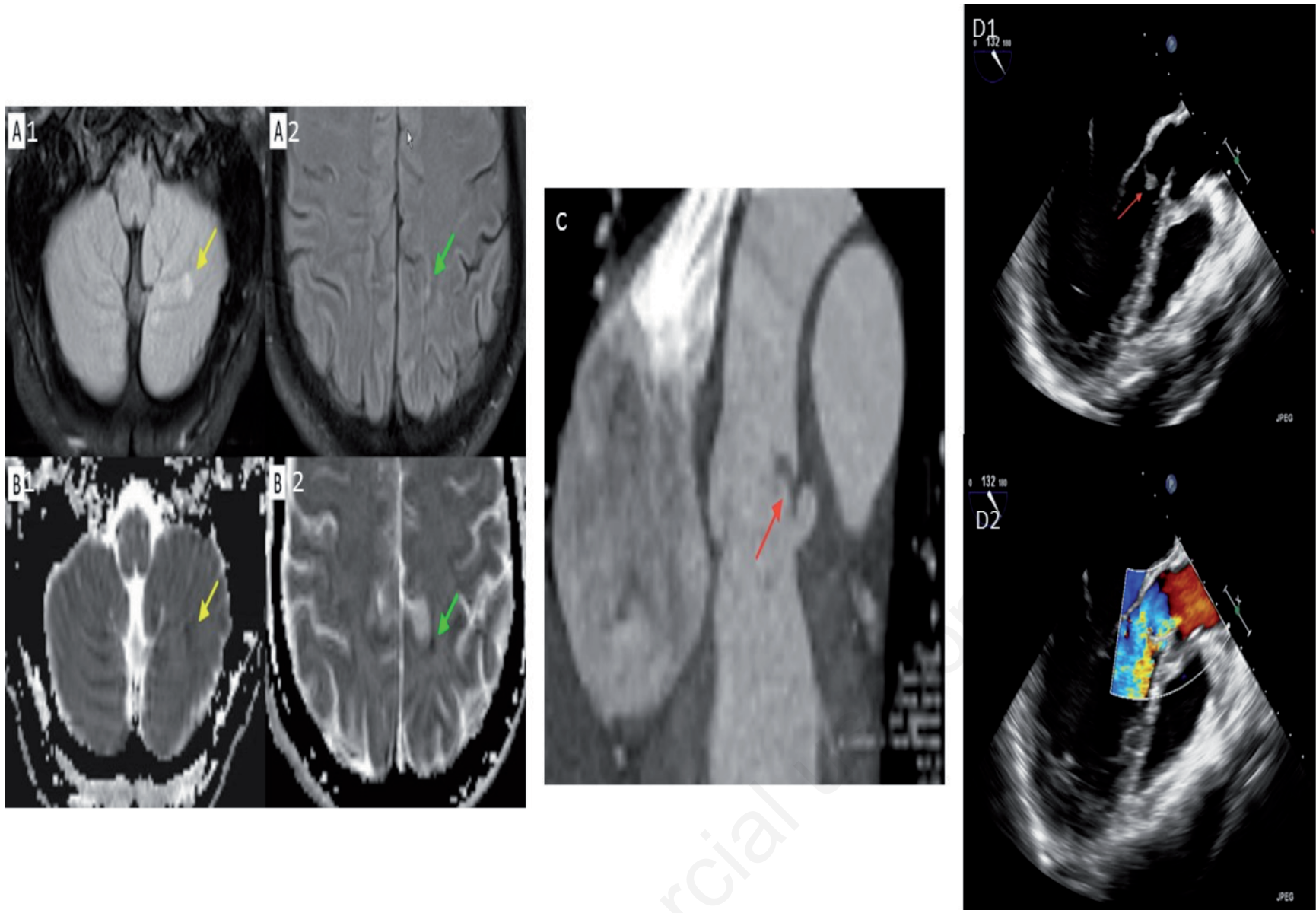

Figure 1. A1, A2, B1, B2) Magnetic resonance imaging of the brain with and without contrast. A) Axial plane: magnetic resonance imaging of the brain showing two foci that are hypointense on apparent diffusion coefficient map and hyperintense on T2/Fluid attenuation inversion recovery in the left cerebellar hemisphere (A) and left parietal lobe (B1). C) CT angiography of the heart demonstrating aortic root erosion or fistula with a diameter of about $4 \mathrm{~mm}$. D1, D2) TEE demonstrating aortic valve vegetation and severe aortic regurgitation.

abuse, underlying medical comorbidities including diabetes mellitus, valvular disease, congenital or acquired spleen dysfunction, and HIV infection can predispose to invasive pneumococcal endocarditis and meningitis $[4,5]$.

Patients with IE are prone to develop hemorrhagic and embolic events. Septic cerebral embolism is reported in around $40 \%$ of patients with IE. IE involving left-sided valves are more prone to develop septic emboli to the brain [6,7]. Risk factors for developing embolic infarcts are older age, prior embolic events, multiple valvular endocarditis, larger vegetation's and virulent organisms like Staphylococcus aureus, and Candida [8,9]. Reports of embolic events in Streptococcus pneumonia are uncommon and mostly in presence of the Austrian syndrome. These embolic infarcts are often multiple, bilateral, and tend to be distributed in the territory of middle cerebral artery territory similar to our patient $[10,11]$. Magnetic Resonance Imaging helps to diagnose, characterize and differentiate acute and subacute infarcts as in our patient. Management is mostly conservative with the role of thrombolysis and mechanical thrombectomy being studied for the suitable patient [12].

The most common site of endocardial involvement in Austrian syndrome is the aortic valve. It is known to have an aggressive course associated with rapid valvular destruction and/or systemic embolism. Aortic root fistula can occur secondary to extension and infiltration of the pyogenic, necrotic tissue. These fistulae can further extend into cardiac chambers, surrounding vasculature including the aorta, coronary arteries, pulmonary artery, and bronchus. These are more common following prosthetic valve-related infections and are better described with cardiac MRI [13-15]. Aortic root fistula secondary to invasive pneumococcal endocarditis is a rare presentation of the same and warrants urgent surgical management like our patient [15].

There has been a report of pneumococcal aortic valve IE presenting with aortopulmonary fistula [16]. As per our knowledge, we report the second patient with the community-acquired pneumococcal, native, aortic valve, endocarditis with Austrian syndrome complicated by the development of an aortic fistula.

\section{References}

1. Aronin SI, Mukherjee SK, West JC, Cooney EL. Review of pneumococcal endocarditis in adults in the penicillin era. Clin Infect Dis 1998;26:165-71.

2. Austrian R. The syndrome of pneumococcal endocarditis, meningitis and rupture of the aortic valve. Trans Am Clin Climatol Assoc 1957;6:40. 
3. Rakočević R, Shapouran S, Pergament KM. Austrian Syndrome - A devastating osler's triad: Case report and literature review. Cureus 2019;11:e4486.

4. Lucas MJ, Brouwer MC, van der Ende A, van de Beek D. 2013. Endocarditis in adults with bacterial meningitis. Circulation 2013;127:2056-62.

5. Mishra AK, Sahu KK, Lal A, Menon V. Aortic valve abscess: staphylococcus epidermidis \& infective endocarditis. QJM 2020;113:211-2.

6. Luzzati R, Pinamonti B, Giacomazzi D, et al. Aortic-right atrium fistula complicating Austrian Syndrome: a case report and literature review. Infez Med 2007;15:124-8.

7. Sahu KK, Mishra AK, Lal A, Kranis M. An interesting case of expressive aphasia: Enterococcus faecalis-related infective endocarditis complicating as septic emboli. QJM 2020;113: 146-7.

8. Mishra AK, Sahu KK, Lal A, Sujata M. Systemic embolization following fungal infective endocarditis. QJM 2020;113: 233-5.

9. Thomas VV, Mishra AK, Jasmine S, Sathyendra S. Gram-negative infective endocarditis: a retrospective analysis of 10 years data on clinical spectrum, risk factor and outcome. Monaldi Arch Chest Dis 2020;90:1359.

10. Mishra AK, Sahu KK, Nagabandi S, Benotti J. Infective endocarditis with mitral leaflet perforation and multiple embolic infarcts. QJM 2020;113:757-9.

11. Mishra AK, Sahu KK, Baddam V, Sargent J. Stroke and infective endocarditis. QJM 2020;113;515-6.

12. Mishra AK, Sahu KK, George AA, Lal A. Safety and efficacy of thrombolysis and mechanical thrombectomy in infective endocarditis. J Stroke Cerebrovasc Dis 2020;29:104784.

13. Mishra AK, Sahu KK, Lal A. Significance of prolonged PR interval in infections. QJM 2020;113:150-1.

14. Sahu KK, Mishra AK, Sherif AA, et al. An interesting case of pacemaker endocarditis. Neth Heart J 2019;27:585-6.

15. Sanchez-Nadales A, Celis-Barreto V, Khan A, et al. Aorto-cavitary fistula to the left ventricle with severe aortic regurgitation as a complication of prosthetic valve infective endocarditis: a novel report. Oxf Med Case Reports 2020;2020:omaa109.

16. Endara A, Corkeron MA, Diqer AM et al. Pneumococcal aortic valve endocarditis causing aortopulmonary artery fistula. Ann Thorac Surg 2001;72:1737-8. 